\title{
Neutron stars: From the inner crust to the core with the (extended) Nambu-Jona-Lasinio model
}

\author{
Helena Pais, ${ }^{1}$ Débora P. Menezes, ${ }^{2}$ and Constança Providência ${ }^{1}$ \\ ${ }^{1}$ CFisUC, Department of Physics, University of Coimbra, P-3004-516 Coimbra, Portugal \\ ${ }^{2}$ Departamento de Física, Universidade Federal de Santa Catarina, Florianópolis, SC, CP 476, CEP 88.040-900, Brazil
}

(Received 22 December 2015; revised manuscript received 11 May 2016; published 8 June 2016)

\begin{abstract}
Nucleonic matter is described within an SU(2) extended Nambu-Jona-Lasinio (NJL) model. Several parametrizations with different nuclear matter saturation properties are proposed. At subsaturation, nuclear pasta phases are calculated within two methods: the coexistence-phases approximation and the compressible liquid drop model, with the surface tension coefficient determined using a geometrical approach at zero temperature. A unified equation of state of stellar matter for the inner crust, with the nuclear pasta phases, and the core is calculated. The mass and radius of neutron stars within this framework are obtained for several families of hadronic and hybrid stars. The quark phase of hybrid stars is described within the SU(3) NJL model including a vector term. Stellar macroscopic properties are in accordance with some of the recent results in the literature.
\end{abstract}

DOI: 10.1103/PhysRevC.93.065805

\section{INTRODUCTION}

Neutron stars still remain today, despite several studies started many years ago, either from an observational point of view or from a theoretical point of view a big question mark, with respect to their constitution and characteristics. Currently, many efforts are being made to try to unravel a bit further our knowledge on these exotic objects, the remnants of core-collapse supernova events (see, e.g., the recent European Physics Journal A topical issue, Exotic Matter in Neutron Stars [1]).

In the inner crust of neutron stars, at subsaturation densities, a frustrated system, named nuclear pasta, appears due to the competition between the Coulomb and the strong forces. These exotic structures have their geometry changing as the density increases. One of the main interests on these nuclear shapes is the effect that they might have on the neutrino transport [2-5] and subsequent cooling of the neutron star. Recent studies [6] have shown evidence on the existence of the pasta phases, where these structures limit the maximum spin period of isolated $\mathrm{x}$-ray pulsars.

The nuclear pasta phase has been studied under different assumptions, namely using semiclassical microscopic treatments, such as quantum molecular dynamics calculations [3,7], or using microscopic calculations, such as 1D Hartree-Fock [8,9] or 3D Hartree-Fock using the SLy4 Skyrme model [10]. Relativistic mean field (RMF) calculations employing a model for the Lagrangian, which is based on microscopic Dirac-Brueckner-Hartree-Fock calculations, using realistic nucleon-nucleon (NN) interactions [11], or using the Thomas-Fermi calculations, based on phenomenological energy-density functionals [12], are also widely used. RMF models do not consider explicit chiral invariance. In this article, we use a different set of models for the description of the nucleonic homogeneous matter and the pasta phase: the extended Nambu-Jona-Lasinio (NJL) model with different parametrizations [13-19], where the chiral symmetry is included. An advantage of these models is the fact that, since they satisfy chiral symmetry, the equation of state (EOS) is also valid at higher densities, such as the ones present in the center of compact objects.
As discussed in [16], a link between QCD and the description of nuclear matter and nuclei through effective hadronic fields can be established including QCD symmetries in the Lagrangian density of the system. Using the extended NJL (eNJL) model developed in the present study, we include chiral symmetry, together with the mechanism of mass generation and binding of nuclear matter, the chiral condensates being built from nucleonic degrees of freedom.

The pasta phase calculation (see, e.g., [20] and references therein) is done by considering two different methods: the coexisting-phases (CP) approximation, where the Gibbs equilibrium conditions are used to get the lowest free-energy state and the surface and Coulomb terms are added "by hand," and the compressible liquid drop (CLD) model, where, unlike the $\mathrm{CP}$ approximation, both the Coulomb and surface terms are taken into account in the minimization of the total energy of the system.

In the present paper, our aim is the complete description of possible matter in the interior of neutron stars. Once matter at subsaturation and suprasaturation densities are obtained and understood within the $\mathrm{SU}(2)$ eNJL, we consider also the SU(3) version of the NJL model [21-25] with a vector interaction [26-29] to describe a possible neutron star core in a hybrid star. We construct the complete stellar EOS by considering the BPS EOS [30] for the outer crust, the pasta EOS for the inner crust, and investigate two different scenarios at high densities: (i) nucleonic matter and (ii) nucleonic and quark matter via Maxwell construction. In the second case, we investigate the possibility of a hybrid star with a quark core. Whenever stellar matter is considered, either in the pasta phase or in its center, $\beta$-equilibrium and charge neutrality are enforced. The procedure just described allows us to obtain the inner crust and the core stellar matter EOS, within the same framework.

The paper is organized as follows: The formalism is briefly reviewed in Sec. II, which is divided into different subsections. We first analyze homogeneous nucleonic matter and its saturation properties obtained with the SU(2) eNJL model and then briefly outline the main aspects of the construction of the pasta phase, including the surface tension calculation. The 
SU(3) NJLv model is then introduced for the description of quark matter, and for the calculation of the hybrid star mass and radius. Section III is devoted to the presentation and discussion of the results, while the main conclusions are given in Sec. IV.

\section{FORMALISM}

In this section, we present the model that describes the nucleonic equation of state (EOS), and make a review of the different formalisms needed to describe the subsaturation and the quark EOS.

\section{A. eNJL model}

The nucleonic NJL model can be extended to yield reasonable saturation properties of nuclear matter, the field $\psi$ being the nucleon field [13-15,17-19]. An effective densitydependent coupling constant is obtained if the following extended NJL (eNJL) Lagrangian density, which actually pushes chiral symmetry restoration to higher densities, is considered:

$$
\begin{aligned}
\mathcal{L}= & \bar{\psi}\left(i \gamma^{\mu} \partial_{\mu}-m\right) \psi+G_{s}\left[(\bar{\psi} \psi)^{2}+\left(\bar{\psi} i \gamma_{5} \vec{\tau} \psi\right)^{2}\right] \\
& -G_{v}\left(\bar{\psi} \gamma^{\mu} \psi\right)^{2}-G_{s v}\left[(\bar{\psi} \psi)^{2}+\left(\bar{\psi} i \gamma_{5} \vec{\tau} \psi\right)^{2}\right]\left(\bar{\psi} \gamma^{\mu} \psi\right)^{2} \\
& -G_{\rho}\left[\left(\bar{\psi} \gamma^{\mu} \vec{\tau} \psi\right)^{2}+\left(\bar{\psi} \gamma_{5} \gamma^{\mu} \vec{\tau} \psi\right)^{2}\right] \\
& -G_{v \rho}\left(\bar{\psi} \gamma^{\mu} \psi\right)^{2}\left[\left(\bar{\psi} \gamma^{\mu} \vec{\tau} \psi\right)^{2}+\left(\bar{\psi} \gamma_{5} \gamma^{\mu} \vec{\tau} \psi\right)^{2}\right] \\
& -G_{s \rho}\left[(\bar{\psi} \psi)^{2}+\left(\bar{\psi} i \gamma_{5} \vec{\tau} \psi\right)^{2}\right]\left[\left(\bar{\psi} \gamma^{\mu} \vec{\tau} \psi\right)^{2}\right. \\
& \left.+\left(\bar{\psi} \gamma_{5} \gamma^{\mu} \vec{\tau} \psi\right)^{2}\right] .
\end{aligned}
$$

For nuclear matter, the degeneracy is $v=2 N_{f}$, and $\Lambda$ is such that $M=939 \mathrm{MeV}$ is the nucleon mass in the vacuum, determined variationally. The term in $G_{v}$ simulates a chiralinvariant short-range repulsion between nucleons. The term in $G_{s v}$ accounts for the density dependence of the scalar coupling. For nuclear matter, the NJL model leads to binding, but the binding energy per particle does not have a minimum except at a rather high density where the nucleon mass is small or vanishing. The introduction of the $G_{s v}$ coupling term is required to correct this. The isovector-vector term (the $G_{\rho}$ term) allows the description of isospin asymmetric nuclear matter. A current mass $m$ term that breaks explicitly the chiral symmetry is introduced in some parametrizations to make the restoration of the chiral symmetry less abrupt. The terms $G_{\omega \rho}$ and $G_{s \rho}$ make the symmetry energy softer.

The thermodynamical potential per volume corresponding to (2) is given by

$$
\begin{aligned}
\omega(\mu)= & \varepsilon_{\text {kin }}+m \rho_{s}-G_{s} \rho_{s}^{2}+G_{v} \rho^{2}+G_{s v} \rho_{s}^{2} \rho^{2}+G_{\rho} \rho_{3}^{2} \\
& +G_{v \rho} \rho^{2} \rho_{3}^{2}+G_{s \rho} \rho_{s}^{2} \rho_{3}^{2}-\mu_{p} \rho_{p}-\mu_{n} \rho_{n},
\end{aligned}
$$

where exchange terms have been neglected. The kinetic energy density is defined as

$$
\begin{aligned}
\varepsilon_{\text {kin }} & =\langle\bar{\psi}(\vec{\gamma} \cdot \vec{p}) \psi\rangle=F_{1}\left(M, k_{F_{i}}\right)-F_{1}(M, \Lambda), \\
F_{1}(M, x) & =\int_{0}^{x} \frac{d p}{\pi^{2}} p^{2} \sqrt{M^{2}+p^{2}}, i=p, n,
\end{aligned}
$$

and $\rho, \rho_{s}$, and $\rho_{3}$ are the baryonic, scalar, and isovector densities, respectively, and are given by $\rho=\rho_{p}+\rho_{n}$, $\rho_{s}=\rho_{s p}+\rho_{s n}$, and $\rho_{3}=\rho_{p}-\rho_{n}$. The proton and neutron densities and scalar densities are given by the usual expressions,

$$
\rho_{i}=\int_{0}^{k_{F_{i}}} \frac{d p}{\pi^{2}} p^{2}
$$

and

$$
\begin{aligned}
\rho_{s_{i}} & =M\left[F_{0}\left(M, k_{F_{i}}\right)-F_{0}(M, \Lambda)\right], \\
F_{0}(M, x) & =\int_{0}^{x} \frac{d p}{\pi^{2}} \frac{p^{2}}{\sqrt{M^{2}+p^{2}}}, \quad i=p, n .
\end{aligned}
$$

The pressure of the system is given by $P=-\omega(\mu)+\varepsilon_{0}$, and the total energy density is given by $\varepsilon=-P+\mu_{p} \rho_{p}+$ $\mu_{n} \rho_{n}$, with $\varepsilon_{0}$ being the energy density in the vacuum. The condition $\partial \omega / \partial M=0$ determines the effective nucleon mass given by

$$
M=m-2 G_{s} \rho_{s}+2 G_{s v} \rho_{s} \rho^{2}+2 G_{s \rho} \rho_{s} \rho_{3}^{2} .
$$

The free nucleon mass, $M_{0}$, is the value of $M$ at zero chemical potential. The conditions $\partial \omega / \partial p_{F_{i}}=0$ determine the chemical potentials,

$$
\begin{aligned}
\mu_{p}= & E_{p_{F}}^{p}+2 G_{v} \rho+2 G_{s v} \rho \rho_{s}^{2}+2 G_{\rho} \rho_{3}+2 G_{v \rho} \rho_{3}^{2} \rho \\
& +2 G_{v \rho} \rho^{2} \rho_{3}+2 G_{s \rho} \rho_{3} \rho_{s}^{2} \\
\mu_{n}= & E_{p_{F}}^{n}+2 G_{v} \rho+2 G_{s v} \rho \rho_{s}^{2}-2 G_{\rho} \rho_{3}+2 G_{v \rho} \rho_{3}^{2} \rho \\
& -2 G_{v \rho} \rho^{2} \rho_{3}-2 G_{s \rho} \rho_{3} \rho_{s}^{2},
\end{aligned}
$$

with $E_{p_{F}}^{i}=\sqrt{M^{2}+p_{F}^{i 2}}, i=p, n$. These conditions together with Eq. (6) fix the values of $p_{F}^{i}, M$ for given $\mu_{i}$.

For reference and to help the discussion, we show in Table I the coupling constants, and in Table II the symmetric nuclear matter properties for the models we are using in this study, eNJLx, eNJLx $\omega \rho y$ and eNJLx $\sigma \rho y$ (without current mass), and eNJLxm and eNJLxm $\sigma \rho y$ (with current mass). Models eNJLx $\omega \rho y$ (eNJLx $\sigma \rho)$ contain the $\omega \rho(\sigma \rho)$ coupling term in the Lagrangian density, i.e., $G_{v \rho} \neq 0\left(G_{s \rho} \neq 0\right)$. We have fixed the symmetry energy at $\rho=0.1 \mathrm{fm}^{-3}$ at the same value obtained for eNJLx (eNJLxm), and we calculated the new $G_{\rho}$ constants, by fixing the $G_{v \rho}\left(G_{s \rho}\right)$ coupling constant.

\section{B. The coexisting-phases approximation and the compressible liquid drop model}

In order to describe the nonuniform npe matter inside the Wigner-Seitz unit cell, which is taken to be a sphere (bubble), a cylinder (tube), or a slab, in three, two, and one dimensions, we use two different methods: the coexistence-phases (CP) approximation and the compressible liquid drop (CLD) model. In the $\mathrm{CP}$ approximation, matter is organized into separated regions of higher and lower density, the higher ones being the pasta phases and the lower ones being a background nucleon gas. The interface between these regions is sharp and finitesize effects are taken into account by surface and Coulomb terms in the energy density [31]. The Gibbs equilibrium conditions are imposed to get the lowest-energy state and, for a temperature $T=T^{I}=T^{I I}$ and a fixed proton fraction, are 
TABLE I. The coupling constants of the models discussed in the present work.

\begin{tabular}{|c|c|c|c|c|c|c|c|c|}
\hline Model & $G_{s}\left(\mathrm{fm}^{2}\right)$ & $G_{v}\left(\mathrm{fm}^{2}\right)$ & $G_{s v}\left(\mathrm{fm}^{8}\right)$ & $G_{\rho}\left(\mathrm{fm}^{2}\right)$ & $G_{v \rho}\left(\mathrm{fm}^{8}\right)$ & $G_{s \rho}\left(\mathrm{fm}^{8}\right)$ & $\Lambda(\mathrm{MeV})$ & $m(\mathrm{MeV})$ \\
\hline eNJL1 & 4.855 & 4.65 & -6.583 & 0.5876 & 0 & 0 & 388.189 & 0 \\
\hline eNJL1 $1 \omega \rho 1$ & 4.855 & 4.65 & -6.583 & 0.5976 & -1 & 0 & 388.189 & 0 \\
\hline eNJL2 & 3.8 & 3.8 & -4.228 & 0.6313 & 0 & 0 & 422.384 & 0 \\
\hline eNJL2 $2 \omega \rho 1$ & 3.8 & 3.8 & -4.228 & 0.6413 & -1 & 0 & 422.384 & 0 \\
\hline eNJL1m & 1.3833 & 1.781 & -2.943 & 0.7 & 0 & 0 & 478.248 & 450 \\
\hline eNJL1m $\sigma \rho 1$ & 1.3833 & 1.781 & -2.943 & 0.0739 & 0 & 1 & 478.248 & 450 \\
\hline eNJL2m & 1.078 & 1.955 & -2.74 & 0.75 & 0 & 0 & 502.466 & 500 \\
\hline eNJL2m $\sigma \rho 1$ & 1.078 & 1.955 & -2.74 & -0.1114 & 0 & 1 & 502.466 & 500 \\
\hline
\end{tabular}

given by

$$
\begin{aligned}
& \mu_{n}^{I}=\mu_{n}^{I I}, \\
& \mu_{p}^{I}=\mu_{p}^{I I}, \\
& P^{I}=P^{I I},
\end{aligned}
$$

where $I$ and $I I$ label the high- and low-density phases, respectively. After the lowest energy state is achieved, the surface and Coulomb terms are added to the total energy density of the system, which is given by

$$
\varepsilon=f \varepsilon^{I}+(1-f) \varepsilon^{I I}+\varepsilon_{e}+\varepsilon_{\text {surf }}+\varepsilon_{\text {Coul }},
$$

where $f$ is the volume fraction of phase $I$.

In the CLD model [32-35], the equilibrium conditions of the system are derived from the minimization of the total free energy [33], including the surface and Coulomb terms. The equilibrium conditions for a fixed proton fraction become

$$
\begin{aligned}
& \mu_{n}^{I}=\mu_{n}^{I I}, \\
& \mu_{p}^{I}=\mu_{p}^{I I}-\frac{\varepsilon_{\text {surf }}}{f(1-f)\left(\rho_{p}^{I}-\rho_{p}^{I I}\right)}, \\
& P^{I}=P^{I I}-\varepsilon_{\text {surf }}\left(\frac{1}{2 \alpha}+\frac{1}{2 \Phi} \frac{\partial \Phi}{\partial f}-\frac{\rho_{p}^{I I}}{f(1-f)\left(\rho_{p}^{I}-\rho_{p}^{I I}\right)}\right),
\end{aligned}
$$

TABLE II. Symmetric nuclear matter properties at saturation density $\rho_{0}$ (energy per particle $B / A$, incompressibility $K$, symmetry energy $E_{\text {sym }}$, and symmetry energy slope $L$ ). All the quantities are in $\mathrm{MeV}$, except for $\rho_{0}$, given in $\mathrm{fm}^{-3}$.

\begin{tabular}{lccccc}
\hline \hline Model & $\rho_{0}$ & $B / A$ & $K$ & $E_{\text {sym }}$ & $L$ \\
\hline eNJL1 & 0.148 & -16.34 & 267.26 & 33.0 & 99.90 \\
eNJL1 $\omega \rho 1$ & 0.148 & -16.34 & 267.26 & 32.65 & 95.02 \\
eNJL1 $\omega \rho 2$ & 0.148 & -16.34 & 267.26 & 30.91 & 70.61 \\
eNJL2 & 0.148 & -15.56 & 231.13 & 33.0 & 95.03 \\
eNJL2 $\omega \rho 1$ & 0.148 & -15.56 & 231.13 & 32.65 & 90.15 \\
eNJL3 & 0.148 & -15.69 & 239.70 & 31.65 & 85.26 \\
eNJL3 $\sigma \rho 1$ & 0.148 & -15.69 & 239.70 & 29.91 & 64.45 \\
eNJL1m & 0.148 & -16.05 & 233.75 & 32.46 & 86.20 \\
eNJL1m $\sigma \rho 1$ & 0.148 & -16.05 & 233.75 & 30.28 & 60.32 \\
eNJL2m & 0.148 & -16.22 & 286.63 & 33.66 & 89.20 \\
eNJL2m $\sigma \rho 1$ & 0.148 & -16.22 & 286.63 & 31.13 & 59.04 \\
\hline \hline
\end{tabular}

where $\alpha=f$ for droplets, rods, and slabs, and $\alpha=1-f$ for tubes and bubbles. $\Phi$ is given by

$$
\Phi= \begin{cases}\left(\frac{2-D \alpha^{1-2 / D}}{D-2}+\alpha\right) \frac{1}{D+2}, & D=1,3, \\ \frac{\alpha-1-\ln \alpha}{D+2}, & D=2 .\end{cases}
$$

For more details on both methods, the reader should refer to [20] and references therein.

\section{The surface tension}

We use a geometrical approach to obtain a numerical value for the surface tension coefficient. This method was introduced and discussed in [36] for quark matter. The surface tension coefficient, $\sigma$, is given by

$$
\sigma=\frac{a}{\rho_{g}}\left(2 \varepsilon_{g}\right)^{1 / 2} \int_{\rho_{1}}^{\rho_{2}}(\Delta \varepsilon)^{1 / 2} d \rho,
$$

with $\rho_{g}=\frac{\rho_{1}+\rho_{2}}{2}, \varepsilon_{g}=\frac{\varepsilon\left(\rho_{1}\right)+\varepsilon\left(\rho_{2}\right)}{2}$, and $\Delta \varepsilon$ the difference between the energy density of homogeneous matter and the nonuniform matter, given by $\Delta \varepsilon=\varepsilon_{h m}-\varepsilon_{n h m}$. These energy densities were fitted to a functional form given by $\varepsilon=b_{0}+b_{1} \rho+b_{2} \rho^{2} . \rho_{1}$ and $\rho_{2}$ are the two coexistence points. In Fig. 1, we show the energy per baryon and the energy density as a function of the density for the homogeneous and nonhomogeneous cases. The surface tension, $\sigma$, which measures the energy per unit area necessary to create a planar interface between the two phases, is defined in terms of the EOS, as in [36]. The width of the interface region and magnitude of $\sigma$ are controlled by the adjustable parameter $a$. Here $a$ was chosen to be 0.1 so that it reproduces the surface tension coefficient for the NL3 model [37] within a ThomasFermi calculation [38], for a fixed proton fraction of $y_{p}=0.5$. We also tested for a different RMF model, the TW model [39], and we obtained a similar result, $a=0.13$, for a fixed proton fraction of 0.5. Since this parameter $a$ depends on the isospin, we calculated it for several values of the proton fraction, and then we fitted it to a functional $a=a_{1}+a_{2} x^{2}+a_{3} x^{4}+a_{4} x^{6}$, in order to calculate the pasta phase in $\beta$-equilibrium matter. We have obtained $a_{1}=-0.00391407, a_{2}=0.251366, a_{3}=$ 5.5648, $a_{4}=-18.5799$, taking NL3 as reference. 

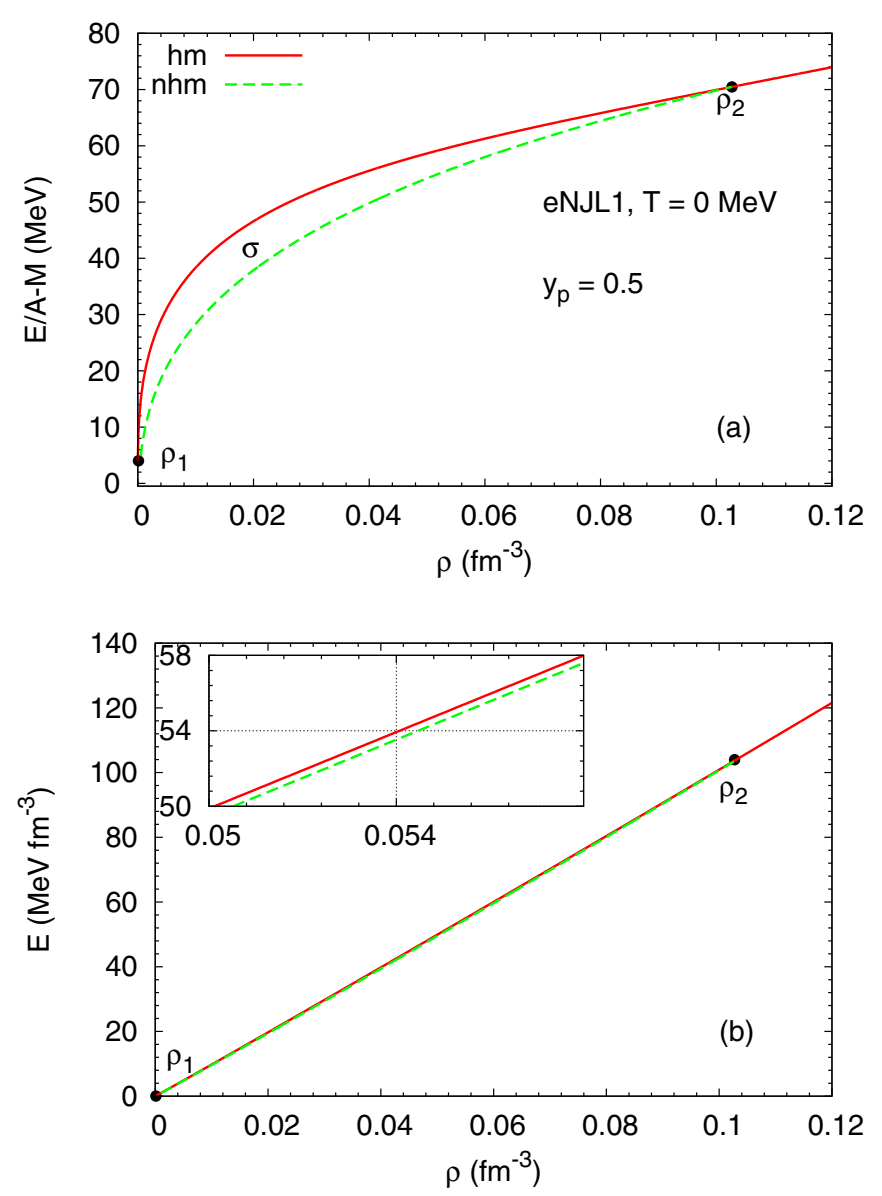

FIG. 1. Energy per baryon (a) and energy density (b) versus density, where the two coexistence points are shown, for homogeneous $(\mathrm{hm})$ and nonhomogeneous (nhm) matter.

\section{NJLv model}

The SU(3) NJL model [21-26] is given by the following Lagrangian density:

$$
\mathcal{L}=\mathcal{L}_{f}+\mathcal{L}_{l}+\mathcal{L}_{\text {sym }}+\mathcal{L}_{\text {vec }}+\mathcal{L}_{\text {det }}
$$

with

$$
\begin{aligned}
\mathcal{L}_{f} & =\bar{\psi}_{f}\left(i \gamma_{\mu} \partial^{\mu}-m_{f}\right) \psi_{f}, \quad \mathcal{L}_{l}=\bar{\psi}_{l}\left(i \gamma_{\mu} \partial^{\mu}-m_{l}\right) \psi_{l}, \\
\mathcal{L}_{\text {sym }} & =G_{s} \sum_{a=0}^{8}\left[\left(\bar{\psi}_{f} \lambda_{a} \psi_{f}\right)^{2}+\left(\bar{\psi}_{f} i \gamma_{5} \lambda_{a} \psi_{f}\right)^{2}\right], \\
\mathcal{L}_{\text {vec }} & =-G_{v} \sum_{a=0}^{8}\left[\left(\bar{\psi}_{f} \gamma^{\mu} \lambda_{a} \psi_{f}\right)^{2}+\left(\bar{\psi}_{f} \gamma^{\mu} \gamma_{5} \lambda_{a} \psi_{f}\right)^{2}\right], \\
\mathcal{L}_{\text {det }} & =-G_{t}\left\{\operatorname{det}_{f}\left[\bar{\psi}_{f}\left(1+\gamma_{5}\right) \psi_{f}\right]+\operatorname{det}_{f}\left[\bar{\psi}_{f}\left(1-\gamma_{5}\right) \psi_{f}\right]\right\} .
\end{aligned}
$$

Here $\psi_{f}$ is the 3 -flavor quark field. The effective mass, $M_{i}$, is given by

$$
M_{i}=m_{i}-4 G_{s} \rho_{s_{i}}-2 G_{t} \rho_{s_{j}} \rho_{s_{k}},
$$

with $(i, j, k)$ being any permutation of $(u, d, s)$, and the chemical potentials by

$$
\mu_{i}=E_{i_{F}}+4 G_{v} \rho_{i}
$$

with $E_{i_{F}}=\sqrt{M_{i}^{2}+p_{F}^{i 2}}, i=u, d, s$. These conditions fix the values of $p_{F}^{i}, M$ for given $\mu_{i}$.

When applying the SU(3) NJLv model to describe the core of hybrid stars in Sec. IIIC, we use a new parametrization, recently proposed in [40], which was built by considering the quark mass in the vacuum equal to $313 \mathrm{MeV}$, and meson properties in the vacuum. This allows both the hadronic and quark models to have the same nucleon mass, while constructing hybrid stars. We consider four different parameter sets, the difference being the strength of the coupling of the vector interaction. The scalar coupling, $G_{s}=1.781 / \Lambda^{2} \mathrm{MeV}^{-2}$, the 't Hooft interaction constant, $G_{t}=-9.29 / \Lambda^{5} \mathrm{MeV}^{-5}$, and the cutoff parameter, $\Lambda=630 \mathrm{MeV}$, are equal for all four parametrizations. The coupling for the vector interaction, $G_{v}$, is given as $G_{v}=x G_{s}, x=0,0.05,0.12,0.2$. We will be calling our set of models NJLi, $i=1,2,3,4$, for $x=0,0.05,0.12,0.2$, respectively.

\section{The Maxwell construction}

We consider different EOS for the construction of a hybrid star: a hadronic EOS, where we use the parameter sets presented in Tables I and II, and a quark EOS, where we consider the NJLi models presented in the first section, and we perform a Maxwell construction. This construction says that two phases are in equilibrium when their chemical potentials, temperatures, and pressures are equal:

$$
\begin{aligned}
T_{H} & =T_{Q}=0, \\
\mu_{H} & =\mu_{Q}, \\
P_{H}(\mu) & =P_{Q}(\mu) .
\end{aligned}
$$

Finding the pressure at the transition, $P_{t}$, will then give us a range of densities with a mixed phase.

With the complete EOS, we integrate the TOV [41,42] equations and find the mass-radius relation for the family of stars. This is a simplified approach to the description of the deconfinement phase transition, but it has been shown that if the surface tension of a quark droplet immersed in nuclear matter is high enough, the results obtained are quite realistic [43].

\section{RESULTS}

In the present section we use the model presented in the previous sections to describe stellar dense matter as found in neutron stars. In particular, we present a unified EOS, except for the outer crust, which is, however, essentially constrained by observational data. We fix the parameters of the model constrained by the properties of nuclear matter at saturation. Properties of symmetric matter and pure neutron matter are discussed. It has been shown that in order to get consistently the radius of stars with masses $M \lesssim 1.5 M_{\odot}$, it is important to describe the inner crust in an appropriate way. We, therefore, calculate the inner crust EOS and discuss how the properties of the EOS affect the structure of the pasta phases. Finally, we build the EOS of $\beta$-equilibrium matter and integrate the TOV equations in order to get the mass versus radius curves. We consider both nucleonic stars and hybrid stars. The EOS 
for hybrid stars takes into account a possible deconfinement phase transition to quark matter. Quark matter is described within the SU(3) NJLi, discussed above, taking into account the possibility of the strangeness onset.

\section{A. Equation of state for symmetric matter}

We consider the parametrizations eNJL1, eNJL2, eNJL3, and eNJL1m and eNJL2m, as referred before, with their parametrizations given in Table I and their properties for symmetric nuclear matter in Table II. For eNJL1, eNJL2, and eNJL3, the symmetry energy properties at saturation are very similar, but their isoscalar properties differ slightly, eNJL2 having a smaller incompressibility. Their values are, however, all within the interval proposed in [44], $K=230 \pm 40 \mathrm{MeV}$. Another property that distinguishes these three models is the density of chiral symmetry restoration, respectively, 0.35 , 0.45 , and $0.998 \mathrm{fm}^{-3}$, for eNJL1, eNJL2, and eNJL3; see Fig. 2, bottom right panel. As we see next, this allows the construction of EOS for hadronic matter with different behaviors at intermediate and high densities.

In Fig. 2, the energy per particle, and pressure (left panels), and the incompressibility and effective mass (right panels) of symmetric nuclear matter are plotted as a function of density. The main feature of these EOS is the change that occurs at the chiral symmetry restoration density, $\rho_{\chi}$ : the EOS becomes much stiffer after the transition, which, for eNJL3, only happens at $\rho \sim 7 \rho_{0}$. Just before the transition, there is a softening clearly seen in the incompressibility, $K$, followed by a change of the slope of the pressure, and a discontinuity
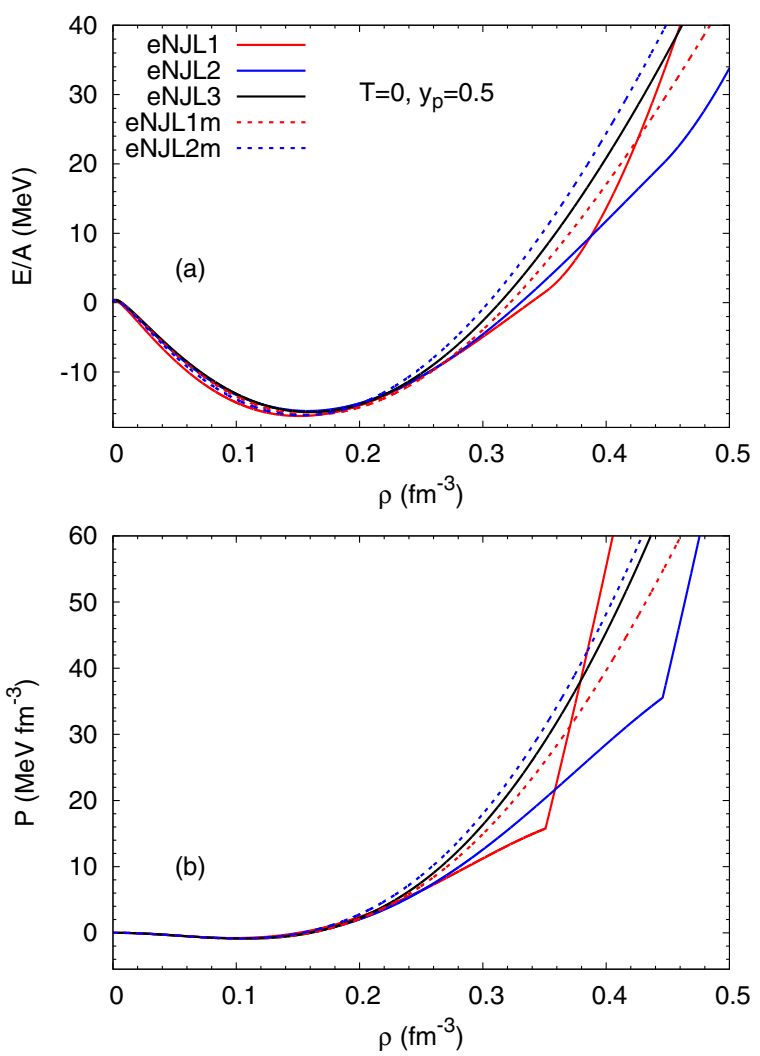

of the incompressibility. The models with current mass do not present this feature. With a proper choice of the parameters, the chiral symmetry restoration takes place at higher densities, and the EOS becomes much softer at intermediate densities. As we will see later, the stiffening of the EOS will allow for very massive stars.

eNJLx and eNJLxm have a quite large slope of the symmetry energy at saturation density, larger than laboratory constraints seem to impose [45]. We, therefore, built six other parametrizations with a smaller slope, $L$, by including in the Lagrangian density a mixed vectorisoscalar-vector-isovector (scalar-isoscalar-vector-isovector) eight point term. These parametrizations are designated by eNJLx $\omega \rho y$ [eNJLx $(m) \sigma \rho y]$. The symmetry energy and its slope at saturation density of all these models are shown as a function of the density in Fig. 3. While below $\rho_{\chi}$, the eNJL1, eNJL2, and eNJL3 models behave as relativistic mean field (RMF) models, see [46,47], above this density, the symmetry energy becomes much softer and its slope may even become immediately negative. This occurs for all models with the mix vector-isoscalar-vector-isovector term. The symmetry energy will eventually become negative at some density above $\rho_{\chi}$, indicating an instability and a tendency for stellar matter to become pure neutron matter. If the restoration of chiral symmetry occurs at a density not high enough, the model becomes inadequate to describe stellar matter. In the last subsection, only models which do not predict a neutron instability will be used to describe stellar matter.

The models with current mass (right panels) do not show such an abrupt behavior as they have a softer restoration
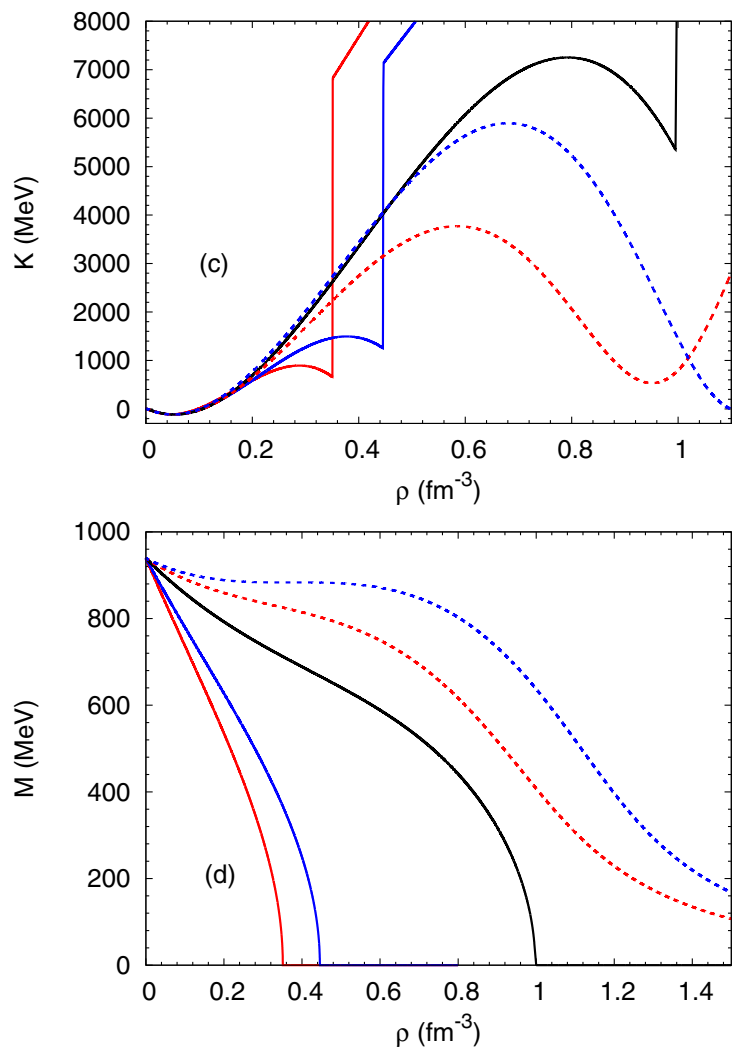

FIG. 2. Symmetric nuclear matter properties as a function of the density for all the models considered in this study. 

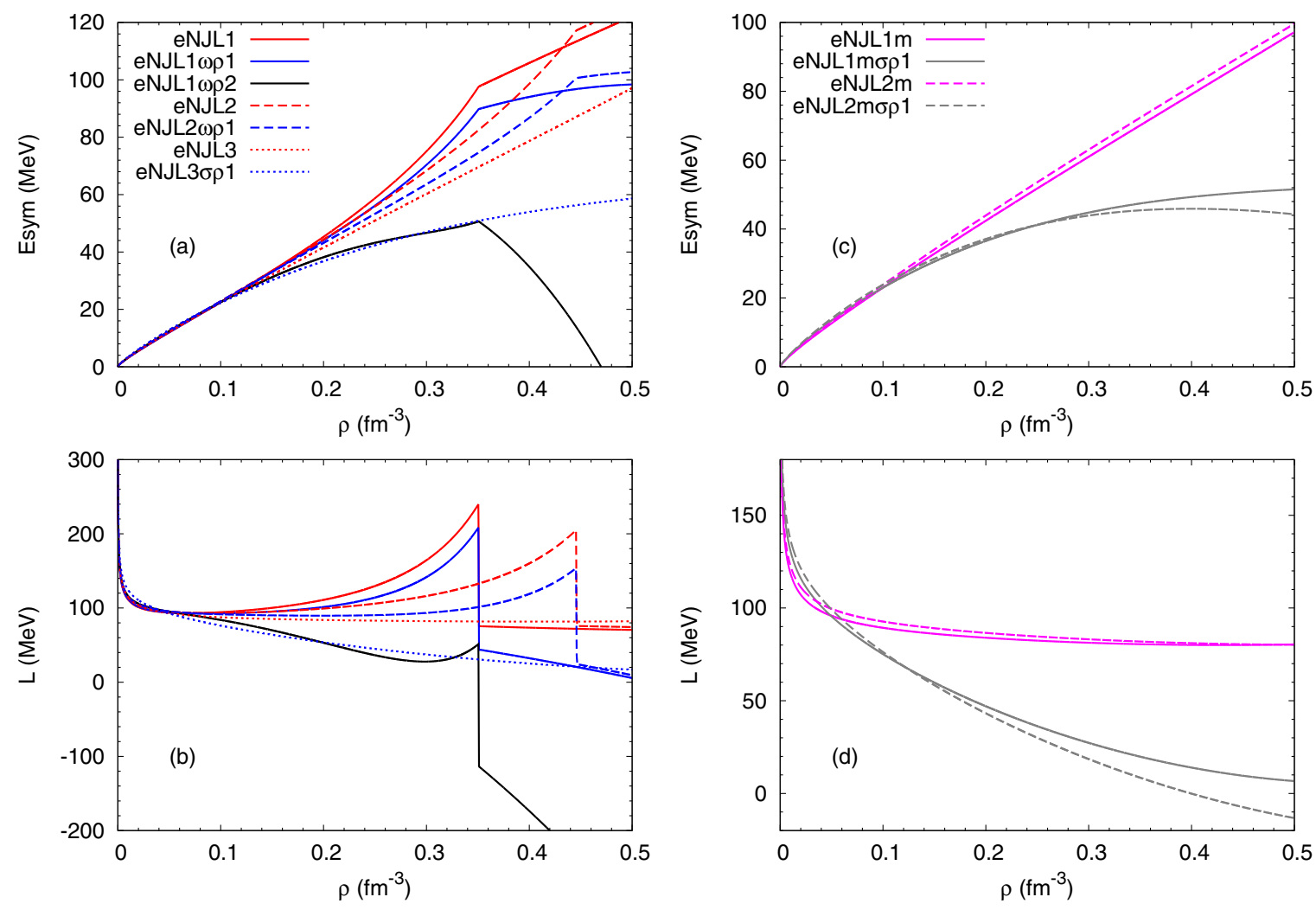

FIG. 3. Symmetry energy, $E_{\text {sym }}$, (a) and (c), and its slope, $L$, (b) and (d), for the models considered in this study.

of the chiral symmetry. For these models, we also consider a term that couples the chiral condensate and the isospin density, to make the symmetry energy less hard, allowing the parametrizations to satisfy constraints from microscopic calculations based on chiral $\mathrm{NN}$ and $3 \mathrm{~N}$ interactions [48] and quantum Monte Carlo results [49]. At the same time, we avoid that the symmetry energy becomes negative below a reasonably high density. This behavior is possible by including a coupling of the isovector-vector term to a isoscalar-scalar term, and not to a isoscalar-vector term, because at high densities the chiral condensate weakens.

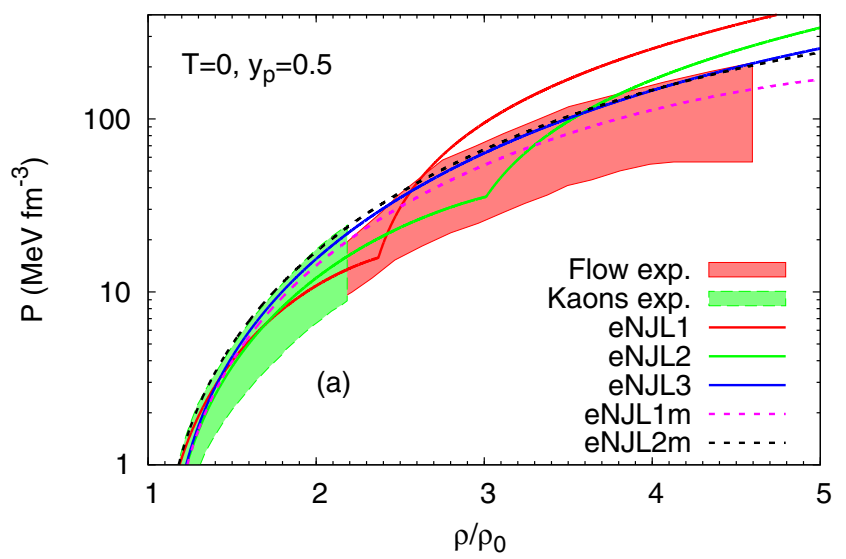

In Fig. 4, we compare the symmetric nuclear matter pressure to experimental results obtained from collective flow data in heavy-ion collisions [50] and from the KaoS experiment [51-54]. The left panel only contains nucleonic EOS. All EOS shown in this panel satisfy the constraints imposed by the KaoS experiment, which refer to densities from above the saturation density to twice the saturation density. The models eNJL2 and eNJL3 are also compatible with constraints from HIC flow experiments. The eNJL1 model, however, becomes quite hard above $2.5 \rho_{0}$, even considering that the constraints imposed in [50] are too

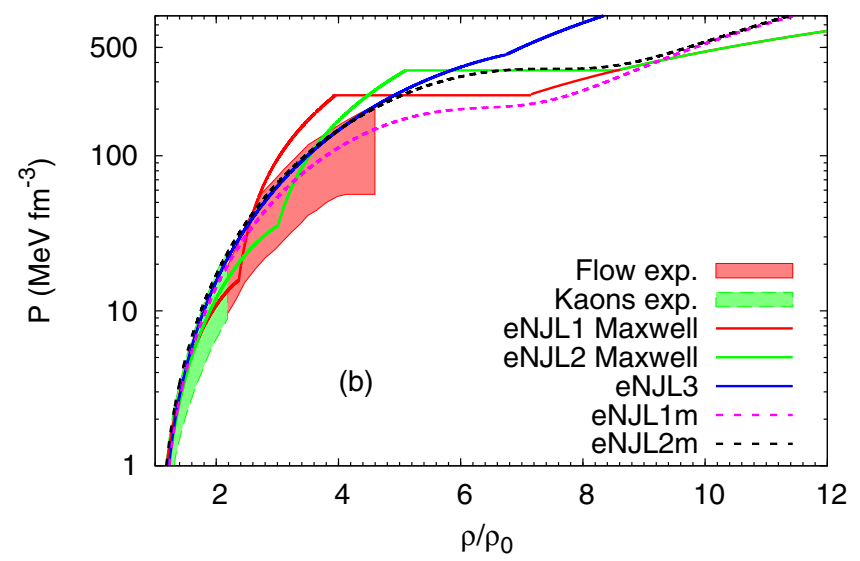

FIG. 4. Pressure as a function of the density for symmetric nuclear matter and for all the models considered in this study. The red region represents the experimental results from Danielewicz et al. [50] and the green region from the KaoS experiment. In the right panel, (b), a Maxwell construction for the eNJL1 and eNJL2 models is also shown. 


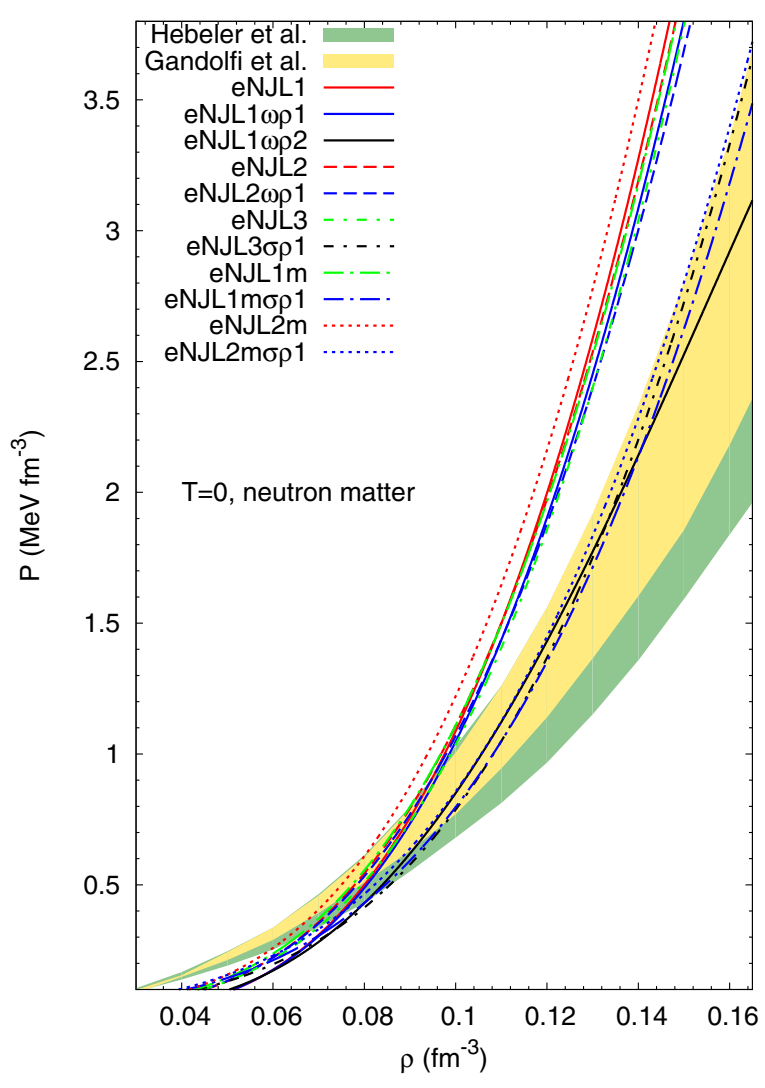

FIG. 5. Pressure as a function of the density for neutron matter for the eNJLx, eNJLx $\omega \rho y$, and eNJLx $(m) \sigma \rho y$ interactions considered in this study. The colored bands are the results from [48] (green) and [49] (gold).

stringent, since the data analysis contains some model dependence.

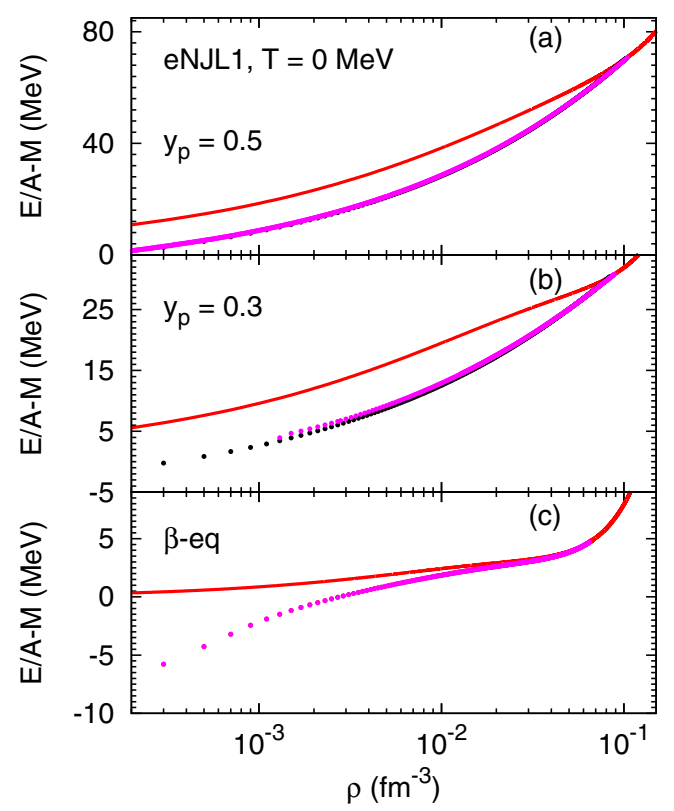

We have allowed for a possible deconfinement phase transition in models eNJL1 and eNJL2. The symmetric matter EOS obtained within a Maxwell construction are plotted in the right panel of Fig. 4. We observe that, for both models, the deconfinement phase transition occurs above the range of densities constrained by the flow data, and, therefore, the conclusions are similar to the ones derived from the left panel. However, for densities above $4 \rho_{0}$, deconfinement gives rise to a clear softening of the EOS. For comparison, we replot in the right panel the EOS of models eNJL1m, eNJL2m, and eNJL3.

We next compare the behavior of neutron matter, calculated with the models under study, with microscopic calculations. In Fig. 5, we show the pressure as a function of the density for pure neutron matter obtained with the eNJLx, eNJLx $\omega \rho y$, and the eNJLxm $\sigma \rho$ models. The two colored bands in the figure are the results from microscopic calculations based on chiral NN and 3N interactions (green) [48] and quantum Monte Carlo results (gold) [49]. In general, almost all models are stiffer than predicted by the microscopic calculations. Only eNJL1 $1 \omega \rho 2$, eNJLxm $\sigma \rho 1$, and eNJL $3 \sigma \rho 1$ models are inside the constrained region for a wide range of densities.

In the following, we will not consider anymore the eNJL1 $\omega \rho 2$ model because, although it presents good properties at saturation and subsaturation densities, at suprasaturation it fails, not only to satisfy heavy-ion flow constraints, but even worse, it predicts a transition to pure neutron matter at a too low density and therefore is not appropriate to describe stellar matter.

\section{B. Subsaturation EOS}

At subsaturation densities, homogeneous nuclear matter is not stable and matter has the tendency to clusterize. Applying the formalisms, CP and CLD, described in Sec. II B, we present in Fig. 6 the energy per particle and the pressure versus density

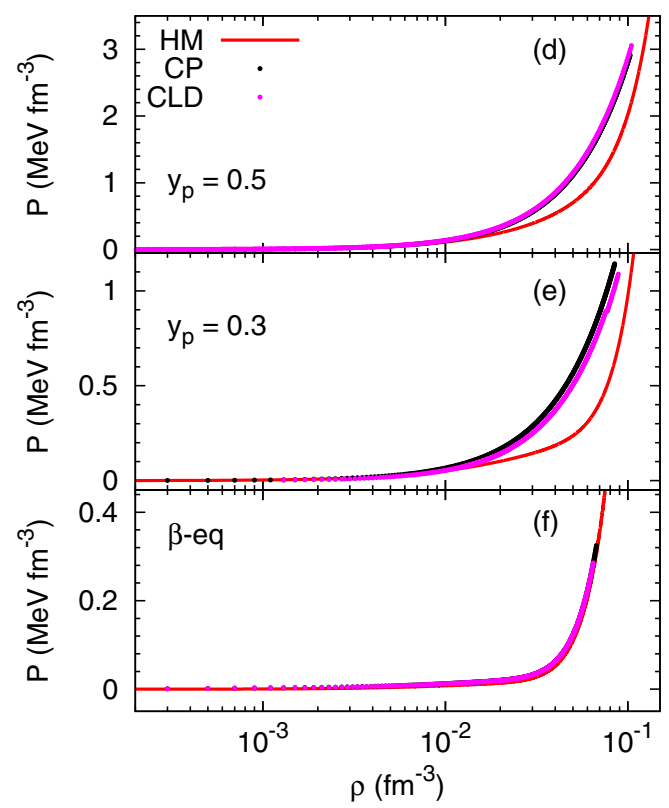

FIG. 6. Energy per baryon, and pressure, as a function of the density for the eNJL1 interaction, for $\beta$-equilibrium matter, (c) and (f), $y_{p}=0.3$, (b) and (e), and $y_{p}=0.5$, (a) and (d), proton fractions. Results with pasta [within the CP (black dots) and CLD (pink dots) approaches] and for homogeneous matter (red solid line) are shown. 

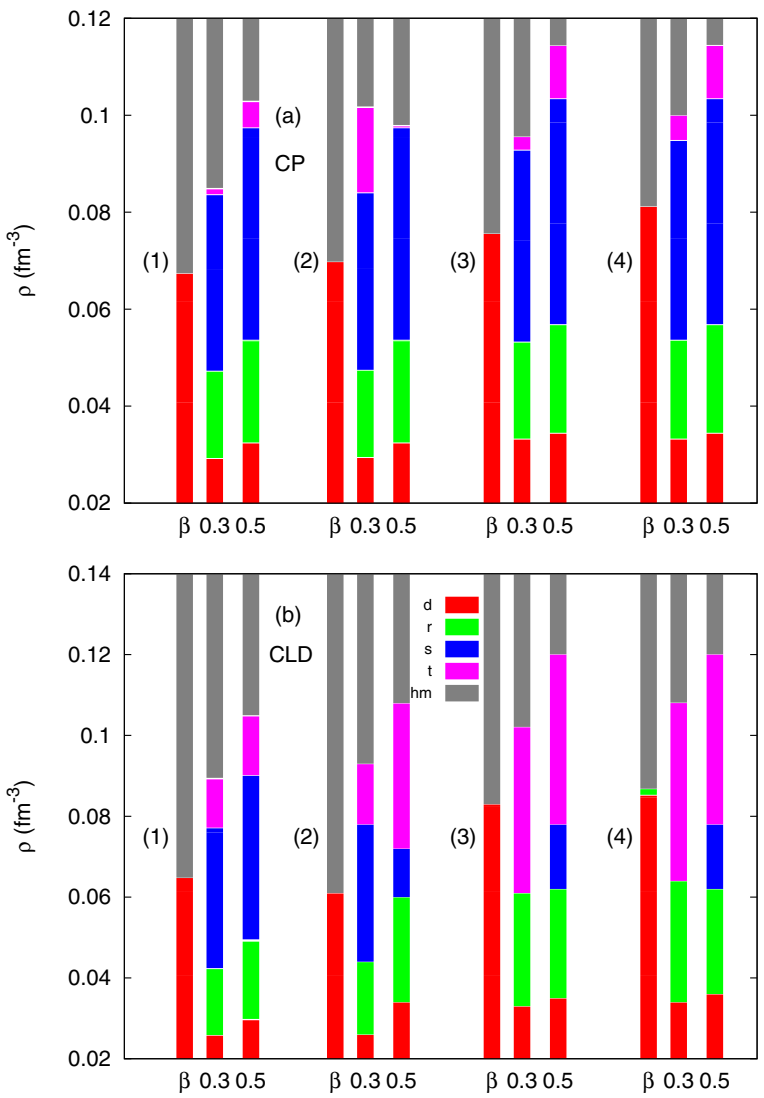

FIG. 7. Pasta phases for the eNJL1 (1), eNJL1 $1 \rho \rho 1$ (2), eNJL3 (3), and eNJL $3 \sigma \rho 1$ (4) interactions within the CP (a) and CLD (b) calculations for $\beta$-equilibrium, $y_{p}=0.3$, and $y_{p}=0.5$ matter. The temperature is fixed to $0 \mathrm{MeV}$.

obtained for homogeneous and clusterized matter, and three different types of matter, $\beta$-equilibrium matter and matter with a proton fraction equal to 0.5 and 0.3 . As expected, clusterized matter has a smaller energy per particle. The CLD and CP methods give similar results, $\mathrm{CP}$ giving rise to a slightly smaller energy per particle due to the fact that the Coulomb field and surface energy contributions are included after the minimization of the free energy. For $y_{p}=0.3$ and 0.5 , there is a first-order phase transition between the clusterized phase and the homogeneous phase.

The different geometries that are present in the nonhomogeneous phase depend on the proton fraction, model, and method. In Fig. 7, the distributions of the different types of shapes as a function of the density are given for four models, eNJL1, eNJL1 $\omega \rho 1$, eNJL3, and eNJL3 $\sigma \rho 1$, and the two methods, CP and CLD. In all models, $\beta$-equilibrium matter does not contain exotic shapes, and clusters are all spherical, independently of model and method, except for a very small region where rods appear for eNJL3 $\sigma \rho 1$. This behavior has been obtained in [55], for models with a large symmetry energy slope at saturation density. In their analysis, $L \lesssim 80 \mathrm{MeV}$ was the condition to obtain other shapes besides droplets.

Matter with $y_{p}=0.3$ and 0.5 has, besides droplets, rods, slabs, and tubes. Bubbles are never present. Generally, the
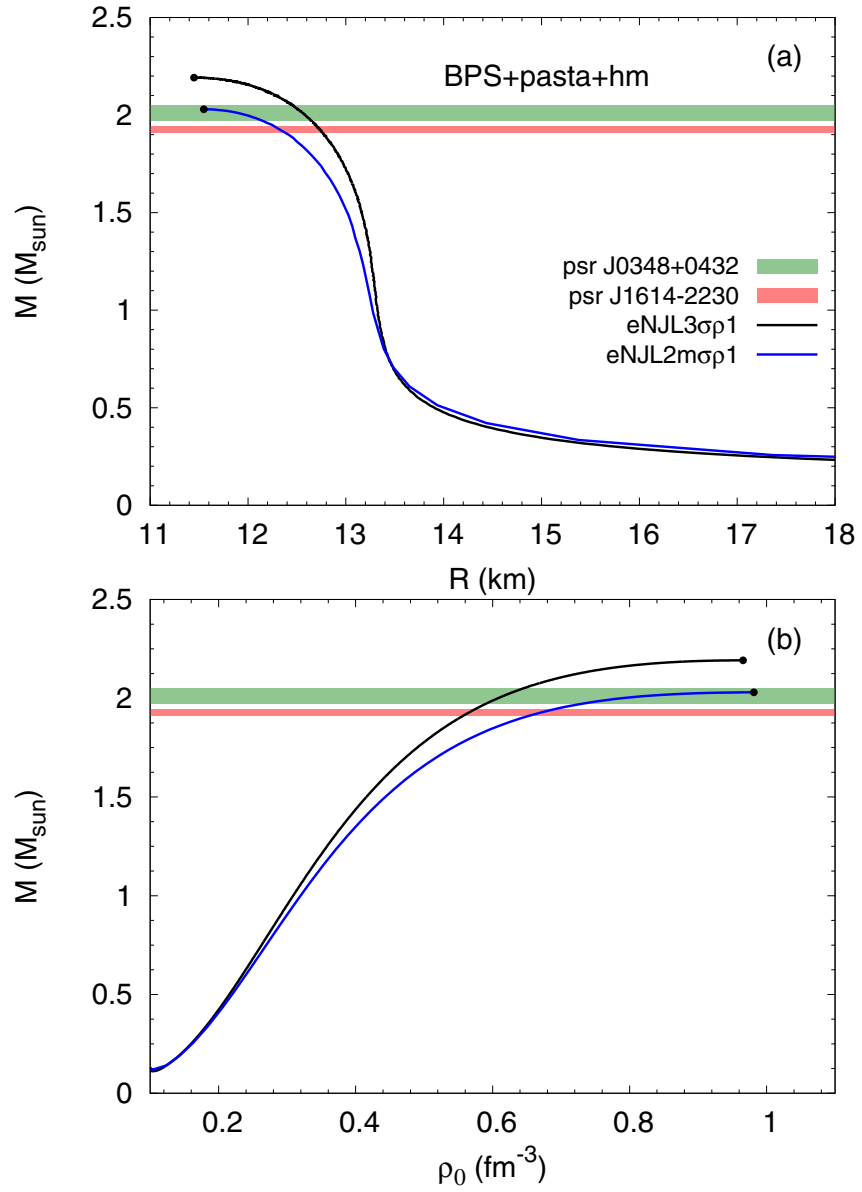

FIG. 8. Mass-radius relation (a) and mass as a function of the central density (b) for the family of hadronic stars that passed the imposed constraints. We have considered pasta in the EOS. The outer crust is given by the BPS EOS. The black dots correspond to the maximum-mass star.

CLD predicts an earlier transition to homogeneous matter and larger tube regions, but this is not always the case.

\section{Neutron stars}

We have built an EOS, appropriate to describe cold stellar matter in $\beta$-equilibrium, as explained in the following: (a) for the outer crust, the Baym-Pethick-Sutherland (BPS) [30] EOS was taken; (b) the inner crust EOS was obtained within the $\mathrm{CP}$ method, and, for reference, we will also show results where the homogeneous matter EOS was taken for densities above neutron drip; (c) the core of nucleonic stars is described using all models, except eNJL1 $\omega \rho 2$, none of which predicts the transition to neutron matter at a density below the central density of the most massive star; (d) for the core of hybrid stars, eNJL1 is considered for the hadronic phase, and the quark phase is described within the SU(3) NJL model.

The $M(R)$ curves of the nucleonic stars are plotted in Figs. 8 and 9, and some of their properties are given in Table III. These properties are the maximum gravitational and baryonic masses and corresponding radii, the central and chiral symmetry restoration densities, the radius of a $1.4 M_{\odot}$ star, and 


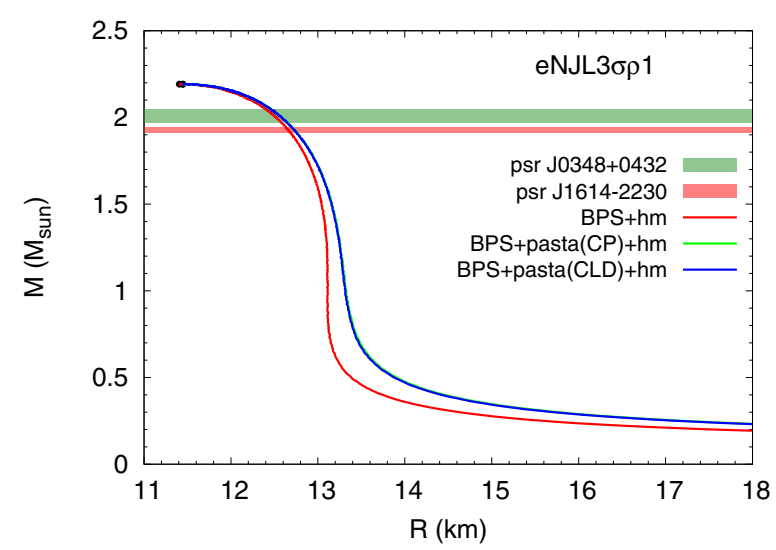

FIG. 9. Mass-radius relation for the eNJL $3 \sigma \rho 1$ model, with pasta from a CP (green) and a CLD (blue) calculations in the EOS, and without it (red). The outer crust is given by the BPS EOS. The black dots correspond to the maximum mass star.

its respective central density. It is seen that the maximum mass and central densities do not depend on how the inner crusts are described, but the radius is sensitive to the crust EOS, a larger radius being obtained when the pasta is included; see Fig. 9 . This difference can be as high as $500 \mathrm{~m}$ for a $1.4 M_{\odot}$ star, and $1 \mathrm{~km}$ for a $1.0 M_{\odot}$ star. The $\beta$-equilibrium EOS of the inner crust, obtained both within the CLD and CP approaches, are very similar, giving rise to almost identical radii, as seen in Fig. 9.

Maximum masses are all above two solar masses, satisfying the constraint imposed by pulsars PSR J0348+0432 [56] and PSR J1614-2230 [57,58]. All models predict a central core of matter for the maximum-mass star with the chiral symmetry restored, except for eNJL3 and eNJL3 $\sigma \rho 1$. However, the $1.4 M_{\odot}$ stars are formed only of matter in the broken phase. Confirming previous results of [59], $1.4 M_{\odot}$ stars have a smaller radius for smaller slopes $L$. Their radii are quite large,
TABLE IV. The models considered and the experimental, observational, and microscopic calculation constraints.

\begin{tabular}{lccccccc}
\hline \hline \multirow{2}{*}{ Model } & \multicolumn{2}{c}{ Experimental } & & \multicolumn{2}{c}{ Microscopic } & \multirow{2}{*}{ Observational } \\
\cline { 2 - 3 } \cline { 7 - 8 } \cline { 7 - 8 } & KaoS & Flow & & $\chi$-NN,3N & QMC & $2 M_{\odot}$ \\
\hline eNJL1 & yes & no & & no & no & yes \\
eNJL1 $\omega \rho 1$ & yes & no & & no & no & yes \\
eNJL1 $\omega \rho 2$ & yes & no & & yes & yes & no \\
eNJL2 & yes & practically & & no & no & yes \\
eNJL2 $\omega \rho 1$ & yes & practically & & no & no & yes \\
eNJL3 & yes & yes & & no & no & yes \\
eNJL3 $\sigma \rho 1$ & yes & yes & & yes & yes & yes \\
eNJL1m & yes & yes & & no & no & yes \\
eNJL1m $\sigma \rho 1$ & yes & yes & & yes & yes & no \\
eNJL2m & yes & yes & & no & no & yes \\
eNJL2m $\sigma \rho 1$ & yes & yes & & yes & yes & yes \\
\hline \hline
\end{tabular}

and the smallest one, obtained including the pasta in the inner crust, is $13.21 \mathrm{~km}$, for a model without current mass in the EOS. The two models with the same value of $L$ have different incompressibilities, and the larger radius corresponds to the larger $K$. Stars within eNJL1 models have larger maximum masses because they have stiffer EOS; see Fig. 2. In the right panels of Fig. 8, we show the $M / R$ relation and mass as a function of the density for the models with current mass in the EOS. eNJL1m $\sigma \rho 1$ is the only one that does not produce a maximum-mass star of $2 M_{\odot}$. All the other models are within the observational constrains.

In Table IV, we show which models satisfy or do not satisfy the experimental and observational constraints and the microscopic calculations ([48,49]).

We have next built a hybrid star EOS, using the Maxwell construction to match the hadronic and quark EOS. Quark matter is described within a new parametrization for the

TABLE III. Some properties of the families of hadronic stars considered. The masses, $M$, the radii, $R$, and the densities, $\rho$, are given in units of $M_{\odot}, \mathrm{km}$, and $\mathrm{fm}^{-3}$, respectively. * These models contain broken chiral symmetry up to the densities considered.

\begin{tabular}{|c|c|c|c|c|c|c|c|}
\hline \multicolumn{8}{|l|}{ with pasta } \\
\hline eNJL1 (CP) & 2.607 & 3.177 & 12.397 & 0.776 & 0.35 & 14.406 & 0.324 \\
\hline eNJL1 $1 \omega \rho 1$ & 2.520 & 3.052 & 12.427 & 0.796 & 0.35 & 14.198 & 0.343 \\
\hline eNJL2 & 2.365 & 2.835 & 11.556 & 0.916 & 0.45 & 14.086 & 0.350 \\
\hline eNJL $3 \sigma \rho 1$ & 2.192 & 2.602 & 11.445 & 0.966 & 0.998 & 13.212 & 0.391 \\
\hline eNJL1m & 2.072 & 2.409 & 12.398 & 0.866 & $*$ & 13.839 & 0.356 \\
\hline eNJL1m $\sigma \rho 1$ & 1.884 & 2.180 & 11.521 & 1.013 & $*$ & 12.809 & 0.449 \\
\hline eNJL2m & 2.275 & 2.683 & 12.380 & 0.846 & $*$ & 14.168 & 0.326 \\
\hline eNJL2m $\sigma \rho 1$ & 2.030 & 2.375 & 11.549 & 0.981 & $*$ & 13.084 & 0.414 \\
\hline \multicolumn{8}{|l|}{ without pasta } \\
\hline eNJL2 $2 \omega \rho 1$ & 2.195 & 2.592 & 11.826 & 0.891 & 0.45 & 13.456 & 0.383 \\
\hline
\end{tabular}


TABLE V. Some properties of the families of hybrid stars considered. The masses, $M$, the radii, $R$, and the densities, $\rho$, are given in units of $M_{\odot}, \mathrm{km}$, and $\mathrm{fm}^{-3}$, respectively. The vector interaction coupling constant, $G_{v}$, for the NJL model is also shown.

\begin{tabular}{|c|c|c|c|c|c|c|c|}
\hline Model & $G_{v} / G_{s}(\mathrm{NJL})$ & $M_{g_{\max }}$ & $M_{b_{\max }}$ & $R$ & $\rho_{c}$ & $R_{M=1.4 M_{\odot}}$ & $\rho_{c_{M=1.4 M_{\odot}}}$ \\
\hline eNJL3 $\sigma \rho 1+$ NJL1 & 0 & 1.795 & 2.051 & 12.069 & 0.923 & 13.211 & 0.392 \\
\hline eNJL3 $\sigma \rho 1+$ NJL2 & 0.05 & 1.884 & 2.166 & 12.386 & 0.840 & 13.211 & 0.392 \\
\hline eNJL3 $\sigma \rho 1+$ NJL4 & 0.2 & 2.074 & 2.430 & 12.378 & 0.671 & 13.211 & 0.392 \\
\hline
\end{tabular}

SU(3) NJL model [40], with a vector interaction, and several strengths of this interaction are considered. Results are presented in Table $\mathrm{V}$ and Fig. 10. We observe that a core of quark matter is obtained for all the cases considered, except for the largest vector coupling (NJL4), where the onset of the quark phase makes the star unstable. This means that stable stars are purely hadronic, or at most, contain in their core a mixed quark-hadron phase. The other three models result in maximum masses below $2 M_{\odot}$, but the one with $G_{v}=0.12 G_{s}$ is above the measured mass $1.928 \pm 0.017 M_{\odot}$ for PSR J1614-2230 [58] and within the limits of the $2.01 \pm 0.04$ $M_{\odot}$ mass of the PSR J0348+0432 [56]. There are other cases where NJL-type models were used for the quark matter EOS, and where massive hybrid stars were found; see, e.g., Ref. [60] and references therein.

\section{CONCLUSIONS}

In the present work, we have described nuclear and stellar matter within a relativistic nuclear model with chiral symmetry. At first, three parametrizations with different isoscalar properties were considered, having different onset densities for the restoration of chiral symmetry. These models present a quite large symmetry energy slope at normal density and, therefore, four other models have been proposed with a smaller slope. To accomplish this new feature, a mixed vectorisovector-vector-isoscalar term, or a mixed scalar-isoscalarvector-isovector term, was included in the Lagrangian density. Above the restoration of chiral symmetry, the EOS of symmetric nuclear matter for all models becomes much stiffer. On the other hand, the symmetry energy softens above the chiral symmetry restoration density and, at large enough densities, it may even become negative, mainly if the modification of the density dependence of the symmetry energy is accomplished by including isovector-vector-isoscalar-vector mixed terms. A special case is the eNJL3, in which the restoration of the chiral symmetry happens at a very high density, $\rho \sim 7 \rho_{0}$. We have also implemented two other models, where we considered a current mass in order to soften the restoration of the chiral symmetry, making the EOS less stiff. A mixed scalar-isoscalar-vector-isovector term was added to the models in order to decrease the slope of the symmetry energy even further, allowing a softening in the EOS.

Neutron star radii are still not well constrained, and it is expected that the future $\mathrm{x}$-ray telescopes, such as NICER and Athena, will impose much stronger constraints. Our results are compatible with some of the present predictions. Both hadronic and hybrid star radii of $1.4 M_{\odot}$ are above $12.8 \mathrm{~km}$, within the observations of the objects BNS $4 \mathrm{U}$ 1608-522 [61], BNS SAX J1748.9-2021 [62,63], and RPMSP PSR J0437-4715 [64,65], but out of the range 10.1-11.1 $\mathrm{km}$ obtained in [66], from the analysis of spectroscopic radius measurements of twelve neutron stars obtained during thermonuclear bursts or in quiescence. However, in [67], it was shown that in order to prevent the EOS from violating causality, the radius should satisfy $R_{1.4} \gtrsim 10.7 \mathrm{~km}$, if it is imposed that the EOS also describes a $2 M_{\odot}$ star. In [45], taking experimental constraints and causality
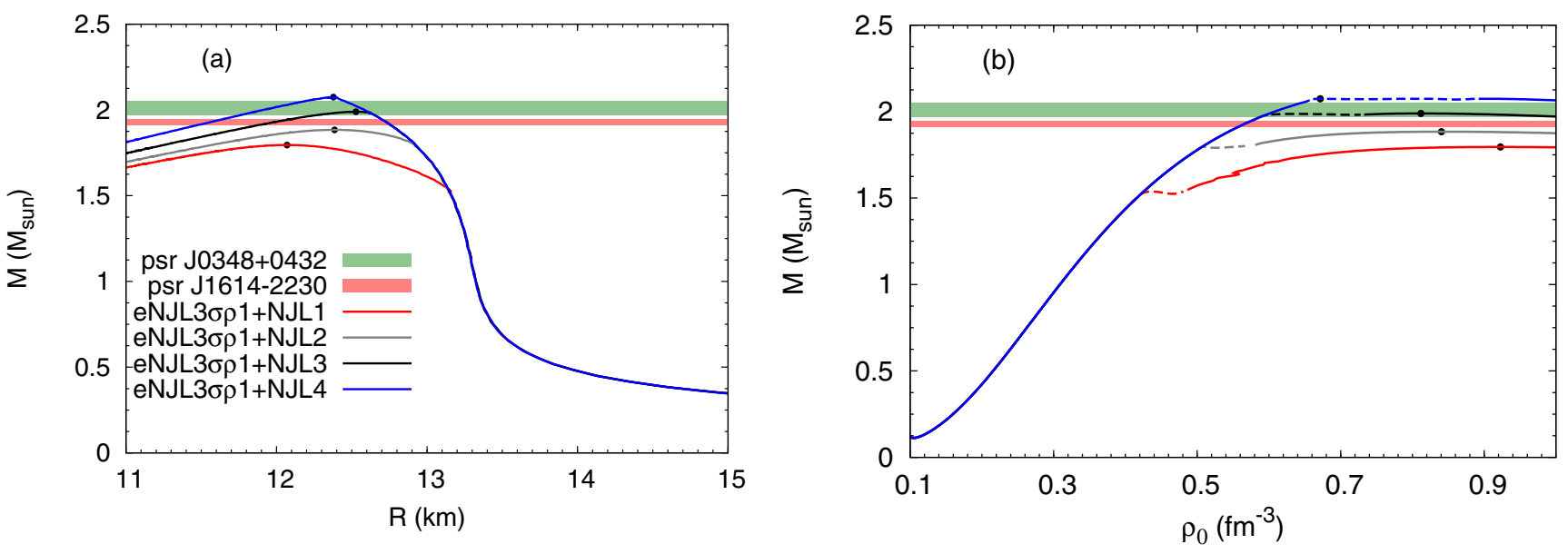

FIG. 10. Mass-radius relation (a) and mass as a function of the central density (b) for the hybrid stars families. Pasta is included in the hadronic EOS. The black dots correspond to the maximum-mass star, and the dashed lines in the left panel correspond to the mixed hadron-quark phase in the stars. 
restrictions for large maximum masses, the $1.4 M_{\odot}$ star radii were constrained to be within the interval $12.1 \pm 1.1 \mathrm{~km}$ (see [68]).

The EOS of nonhomogeneous subsaturation matter was built within the CP and the CLD methods, and we found that within these models, $\beta$-equilibrium matter does not present a nonspherical pasta phase, except for eNJL3 $\sigma \rho 1$. Nonspherical shapes will, however, occur for larger proton fractions, and could exist in core-collapse supernova matter. However, to describe this kind of matter, a finite-temperature calculation must be performed.

Having the subsaturation EOS of $\beta$-equilibrium matter, we have built an almost unified hadronic stellar matter EOS, with the outer crust described by the BPS EOS, and the inner crust and core described within the eNJL model. It was shown that an uncertainty of 0.5 and $1 \mathrm{~km}$ in the radius, respectively, of a 1.4 and a $1.0 M_{\odot}$ star is obtained when the homogeneous matter EOS is used to describe the inner crust.

For the core, we have considered not only nucleonic matter, but also a possible phase transition to quark matter, described within the SU(3) NJL model. In the quark model, we have included a vector term that allows us to turn the quark EOS stiffer. For all nucleonic star families obtained with the models that do not predict a neutron instability for densities below the central density, the maximum mass obtained is well above $2 M_{\odot}$. The inclusion of a possible deconfinement phase transition either decreases the maximum mass (as expected) to values below $2 M_{\odot}$, but still within the mass constraints imposed by the pulsars PSR J1614-2230 [57,58] and PSR J0348+0432 [56], or renders the star with a quark core unstable.

\section{ACKNOWLEDGMENTS}

H.P. is supported by FCT under Project No. SFRH/BPD/95566/2013. She is very thankful to D.P.M. and the Departamento de Física of the Universidade Federal de Santa Catarina for the kind hospitality during her stay there. She is also grateful to M. B. Pinto and F. Gulminelli for useful discussions. Fruitful discussions with Pedro Costa are acknowledged. D.P.M. acknowledges support by CNPq (Grant No. 300602/2009-0) and FAPESC (Brazil) under Project No. 2716/2012, TR 2012000344. Partial support comes from "NewCompStar," COST Action MP1304.
[1] D. Blaschke, J. Schaffner-Bielich, and H.-J. Schulze (eds.), Exotic Matter in Neutron Stars, topical issue of Eur. Phys. J. A 52(3) (2016).

[2] C. J. Horowitz, M. A. Pérez-García, and J. Piekarewicz, Phys. Rev. C 69, 045804 (2004).

[3] C. J. Horowitz, M. A. Pérez-García, J. Carriere, D. K. Berry, and J. Piekarewicz, Phys. Rev. C 70, 065806 (2004).

[4] H. Sonoda, G. Watanabe, K. Sato, T. Takiwaki, K. Yasuoka, and T. Ebisuzaki, Phys. Rev. C 75, 042801(R) (2007).

[5] M. D. Alloy and D. P. Menezes, Phys. Rev. C 83, 035803 (2011).

[6] J. A. Pons, D. Viganò, and N. Rea, Nat. Phys. 9, 431 (2013).

[7] G. Watanabe, H. Sonoda, T. Maruyama, K. Sato, K. Yasuoka, and T. Ebisuzaki, Phys. Rev. Lett. 103, 121101 (2009).

[8] P. Bonche and D. Vautherin, Nucl. Phys. A 372, 496 (1981).

[9] P. Bonche and D. Vautherin, Astron. Astrophys. 112, 268 (1982).

[10] P. Magierski and P.-H. Heenen, Phys. Rev. C 65, 045804 (2002).

[11] P. Gögelein, E. N. E. van Dalen, C. Fuchs, and H. Müther, Phys. Rev. C 77, 025802 (2008).

[12] S. S. Avancini, D. P. Menezes, M. D. Alloy, J. R. Marinelli, M. M. W. Moraes, and C. Providência, Phys. Rev. C 78, 015802 (2008).

[13] V. Koch, T. S. Biro, J. Kunz, and U. Mosel, Phys. Lett. B 185, 1 (1987).

[14] W. Bentz and A. W. Thomas, Nucl. Phys. A 696, 138 (2001).

[15] S. A. Moszkowski, C. Providência, and J. da Providência, in 150 Years of Quantum Many-Body Theory (World Scientific, Singapore, 2001), pp. 197-202.

[16] T. J. Bürvenich and D. G. Madland, Nucl. Phys. A 729, 769 (2003).

[17] C. Providência, J. M. Moreira, J. da Providência, and S. A. Moszkowski, in Hadron Physics: Effective Theories of Low Energy QCD, AIP Conf. Ser., Vol. 660, edited by A. H. Blin, B. Hiller, A. A. Osipov, M. C. Ruivo, and E. van Beveren (AIP, Melville, NY, 2003), pp. 231-241.
[18] I. N. Mishustin, L. M. Satarov, and W. Greiner, Phys. Rep. 391, 363 (2004).

[19] S. A. Moszkowski, C. da Providência, J. da Providência, and J. M. Moreira, arXiv:nucl-th/0204047.

[20] H. Pais, S. Chiacchiera, and C. Providência, Phys. Rev. C 91, 055801 (2015).

[21] Y. Nambu and G. Jona-Lasinio, Phys. Rev. 122, 345 (1961).

[22] Y. Nambu and G. Jona-Lasinio, Phys. Rev. 124, 246 (1961).

[23] U. Vogl and W. Weise, Prog. Part. Nucl. Phys. 27, 195 (1991).

[24] S. P. Klevansky, Rev. Mod. Phys. 64, 649 (1992).

[25] M. Buballa, Phys. Rep. 407, 205 (2005).

[26] D. P. Menezes, M. B. Pinto, L. B. Castro, P. Costa, and C. Providência, Phys. Rev. C 89, 055207 (2014).

[27] M. Hanauske, L. M. Satarov, I. N. Mishustin, H. Stöcker, and W. Greiner, Phys. Rev. D 64, 043005 (2001).

[28] G. Pagliara and J. Schaffner-Bielich, Phys. Rev. D 77, 063004 (2008).

[29] L. Bonanno and A. Sedrakian, Astron. Astrophys. 539, A16 (2012).

[30] G. Baym, C. Pethick, and P. Sutherland, Astrophys. J. 170, 299 (1971)

[31] S. S. Avancini, C. C. Barros, Jr., L. Brito, S. Chiacchiera, D. P. Menezes, and C. Providência, Phys. Rev. C 85, 035806 (2012).

[32] J. M. Lattimer and F. D. Swesty, Nucl. Phys. A 535, 331 (1991).

[33] G. Baym, H. A. Bethe, and C. J. Pethick, Nucl. Phys. A 175, 225 (1971).

[34] J. M. Lattimer, C. J. Pethick, D. G. Ravenhall, and D. Q. Lamb, Nucl. Phys. A 432, 646 (1985).

[35] S. S. Bao, J. N. Hu, Z. W. Zhang, and H. Shen, Phys. Rev. C 90, 045802 (2014).

[36] M. B. Pinto, V. Koch, and J. Randrup, Phys. Rev. C 86, 025203 (2012).

[37] G. A. Lalazissis, J. König, and P. Ring, Phys. Rev. C 55, 540 (1997). 
[38] S. S. Avancini, C. C. Barros, Jr., D. P. Menezes, and C. Providência, Phys. Rev. C 82, 025808 (2010).

[39] S. Typel and H. H. Wolter, Nucl. Phys. A 656, 331 (1999).

[40] R. Pereira, P. Costa, and C. Providência (unpublished).

[41] R. C. Tolman, Phys. Rev. 55, 364 (1939).

[42] J. R. Oppenheimer and G. M. Volkoff, Phys. Rev. 55, 374 (1939).

[43] N. Yasutake, R. Łastowiecki, S. Benić, D. Blaschke, T. Maruyama, and T. Tatsumi, Phys. Rev. C 89, 065803 (2014).

[44] E. Khan, N. Paar, D. Vretenar, L.-G. Cao, H. Sagawa, and G. Colò, Phys. Rev. C 87, 064311 (2013).

[45] A. W. Steiner, J. M. Lattimer, and E. F. Brown, Eur. Phys. J. A 52, 18 (2016).

[46] C. Ducoin, C. Providência, A. M. Santos, L. Brito, and P. Chomaz, Phys. Rev. C 78, 055801 (2008).

[47] C. Ducoin, J. Margueron, C. Providência, and I. Vidaña, Phys. Rev. C 83, 045810 (2011).

[48] K. Hebeler, J. M. Lattimer, C. J. Pethick, and A. Schwenk, Astrophys. J. 773, 11 (2013).

[49] S. Gandolfi, J. Carlson, and S. Reddy, Phys. Rev. C 85, 032801 (2012).

[50] P. Danielewicz, R. Lacey, and W. G. Lynch, Science 298, 1592 (2002).

[51] W. G. Lynch, M. B. Tsang, Y. Zhang, P. Danielewicz, M. Famiano, Z. Li, and A. W. Steiner, Prog. Part. Nucl. Phys. 62, 427 (2009).

[52] C. Fuchs, Prog. Part. Nucl. Phys. 56, 1 (2006).

[53] I. Sagert, L. Tolos, D. Chatterjee, J. Schaffner-Bielich, and C. Sturm, Phys. Rev. C 86, 045802 (2012).

[54] C. Fuchs, A. Faessler, E. Zabrodin, and Y. M. Zheng, Phys. Rev. Lett. 86, 1974 (2001).

[55] K. Oyamatsu and K. Iida, Phys. Rev. C 75, 015801 (2007).

[56] J. Antoniadis, P. C. C. Freire, N. Wex, T. M. Tauris, R. S. Lynch, M. H. van Kerkwijk, M. Kramer, C. Bassa, V. S. Dhillon,
T. Driebe, J. W. T. Hessels, V. M. Kaspi, V. I. Kondratiev, N. Langer, T. R. Marsh, M. A. McLaughlin, T. T. Pennucci, S. M. Ransom, I. H. Stairs, J. van Leeuwen, J. P. W. Verbiest, and D. G. Whelan, Science 340, 448 (2013).

[57] P. B. Demorest, T. Pennucci, S. M. Ransom, M. S. E. Roberts, and J. W. T. Hessels, Nature (London) 467, 1081 (2010).

[58] E. Fonseca, T. T. Pennucci, J. A. Ellis, I. H. Stairs, D. J. Nice, S. M. Ransom, P. B. Demorest, Z. Arzoumanian, K. Crowter, T. Dolch, R. D. Ferdman, M. E. Gonzalez, G. Jones, M. L. Jones, M. T. Lam, L. Levin, M. A. McLaughlin, K. Stovall, J. K. Swiggum, and W. Zhu, arXiv:1603.00545.

[59] J. Carriere, C. J. Horowitz, and J. Piekarewicz, Astrophys. J. 593, 463 (2003).

[60] S. Benić, D. Blaschke, D. E. Alvarez-Castillo, T. Fischer, and S. Typel, Astron. Astrophys. 577, A40 (2015).

[61] J. Poutanen, J. Nättilä, J. J. E. Kajava, O.-M. Latvala, D. K. Galloway, E. Kuulkers, and V. F. Suleimanov, Mon. Not. R. Astron. Soc. 442, 3777 (2014).

[62] T. Güver and F. Özel, Astrophys. J. Lett. 765, L1 (2013).

[63] C. O. Heinke, H. N. Cohn, P. M. Lugger, N. A. Webb, W. C. G. Ho, J. Anderson, S. Campana, S. Bogdanov, D. Haggard, A. M. Cool, and J. E. Grindlay, Mon. Not. R. Astron. Soc. 444, 443 (2014).

[64] S. Bogdanov, Astrophys. J. 762, 96 (2013).

[65] J. P. W. Verbiest, M. Bailes, W. van Straten, G. B. Hobbs, R. T. Edwards, R. N. Manchester, N. D. R. Bhat, J. M. Sarkissian, B. A. Jacoby, and S. R. Kulkarni, Astrophys. J. 679, 675 (2008).

[66] F. Özel, D. Psaltis, T. Güver, G. Baym, C. Heinke, and S. Guillot, Astrophys. J. 820, 28 (2016).

[67] W.-C. Chen and J. Piekarewicz, Phys. Rev. Lett. 115, 161101 (2015)

[68] M. Fortin, J. L. Zdunik, P. Haensel, and M. Bejger, Astron. Astrophys. 576, A68 (2015). 